\title{
Fine lattice simulations with the Ginsparg-Wilson fermions
}

\section{J. Noaki ${ }^{* a} \dagger$, S. Aoki ${ }^{b, c}$, G. Cossu ${ }^{a}$, H. Fukaya ${ }^{d}$, S. Hashimoto ${ }^{a, e}$, T. Kaneko ${ }^{a, e}$ for JLQCD Collaboration}

${ }^{a}$ High Energy Accelerator Research Organization (KEK), Tsukuba 305-0801, Japan

${ }^{b}$ Graduate School of Pure and Applied Sciences, University of Tsukuba, Tsukuba 305-8571, Japan

${ }^{c}$ Yukawa Institute for Theoretical Physics, Kyoto University, Kyoto 606-8502, Japan

${ }^{d}$ Department of Physics, Osaka University Toyonaka, Osaka 560-0043, Japan

e School of High Energy Accelerator Science, the Graduate University for Advanced Studies (Sokendai), Tsukuba 305-0801, Japan

\begin{abstract}
We present the status of 2+1-flavor QCD simulations with the Möbius domain-wall fermions at lattice spacings of $a^{-1}=2.4$ and $3.6 \mathrm{GeV}$. Violation of the Ginsparg-Wilson relation measured by the residual mass is below $0.5 \mathrm{MeV}$ or lower. After giving an overview of the gauge ensemble generation, we discuss the scale setting by the Yang-Mills flow observables in detail. We also discuss autocorrelations in the hybrid Monte Carlo simulations and correlations between lighthadron correlators and the topological charge.
\end{abstract}

The 32nd International Symposium on Lattice Field Theory

23-28 June, 2014

Columbia University New York, NY

\footnotetext{
* Speaker.

${ }^{\dagger}$ Email: noaki@ post.kek.jp
} 


\section{Overview of numerical simulation}

We are aiming at performing precise lattice QCD calculations to test the heavy-flavor sector of the Standard Model. In order to achieve a good control of systematic errors, we generate $N_{f}=2+1$ gauge configurations using the Möbius domain-wall fermion action with the kernel built after three levels of the stout-smearing. The tree-level improved Symanzik action is employed for the gauge sector. The formalism and the status at an earlier stage were presented in [W]. For the development of our simulation code, see []. We designed the numerical simulation to have:

- multiple lattice spacings at $a^{-1}=2.4 \mathrm{GeV}$ and finer, which allow continuum extrapolation of heavy-quark matrix elements with discretization errors under control.

- good chiral property of the light-quark sector, which allows a precise chiral extrapolation without introducing extra terms due to discretization effects. Good control of the operator mixing between different chiralities is also achieved.

- large enough physical volume to suppress finite size effects. Most of the ensembles satisfy the condition $m_{\pi} L \gtrsim 4.0$.

The target lattice spacings are $a^{-1} \approx 2.4,3.6$ and $4.6 \mathrm{GeV}$. Besides the finest one which is in progress, the gauge ensembles have been generated at three values of the up and down quark masses $m_{\mathrm{ud}}$ corresponding to $m_{\pi} \approx 500,400$ and $300 \mathrm{MeV}$. We take two values of the strange quark mass $m_{\mathrm{s}}$ to allow interpolation to the physical mass point. At $a^{-1} \approx 2.4 \mathrm{GeV}$, we also have two ensembles with $m_{\pi} \approx 240 \mathrm{MeV}$ in different volumes. A comparison of these ensembles will

\begin{tabular}{cccccccl}
\hline$\beta$ & $a^{-1}[\mathrm{GeV}]$ & $L_{5}$ & size & $m_{\mathrm{s}}$ & $m_{\mathrm{ud}}$ & $m_{\pi}[\mathrm{MeV}]$ & \#trajs \\
\hline 4.17 & 2.4 & 12 & $32^{3} \times 64$ & 0.030 & 0.0070 & 313 & $10,000(\tau=1)$ \\
& & & & & 0.0120 & 406 & 10,000 \\
& & & & 0.0190 & 508 & 10,000 \\
\hline & & & $32^{3} \times 64$ & 0.040 & 0.0035 & 234 & $10,000(\tau=1)$ \\
& & & & 0.0070 & 315 & 10,000 \\
& & & & 0.0120 & 407 & 10,000 \\
& & & & 0.0190 & 507 & 10,000 \\
\hline \hline 4.35 & 3.6 & 8 & $48^{3} \times 96$ & 0.018 & 0.0042 & 299 & $5,000(\tau=2)$ \\
& & & & & 0.0080 & 415 & 5,000 \\
& & & & & 0.0120 & 505 & 5,000 \\
\hline & & & $48^{3} \times 96$ & 0.025 & 0.0042 & 300 & $5,000(\tau=2)$ \\
& & & & & 0.0080 & 411 & 5,000 \\
& & & & & 0.0120 & 508 & 5,000 \\
\hline \hline 4.47 & 4.6 & 8 & $64^{3} \times 128$ & 0.015 & 0.0060 & $\approx 300$ & $1,425(\tau=4)$ \\
\hline & & & & & & & \\
\hline
\end{tabular}

Table 1: Gauge ensembles. The second column lists the target lattice spacing. The last column shows the number of thermalized trajectories in unit of the molecular dynamics time $\tau$. 

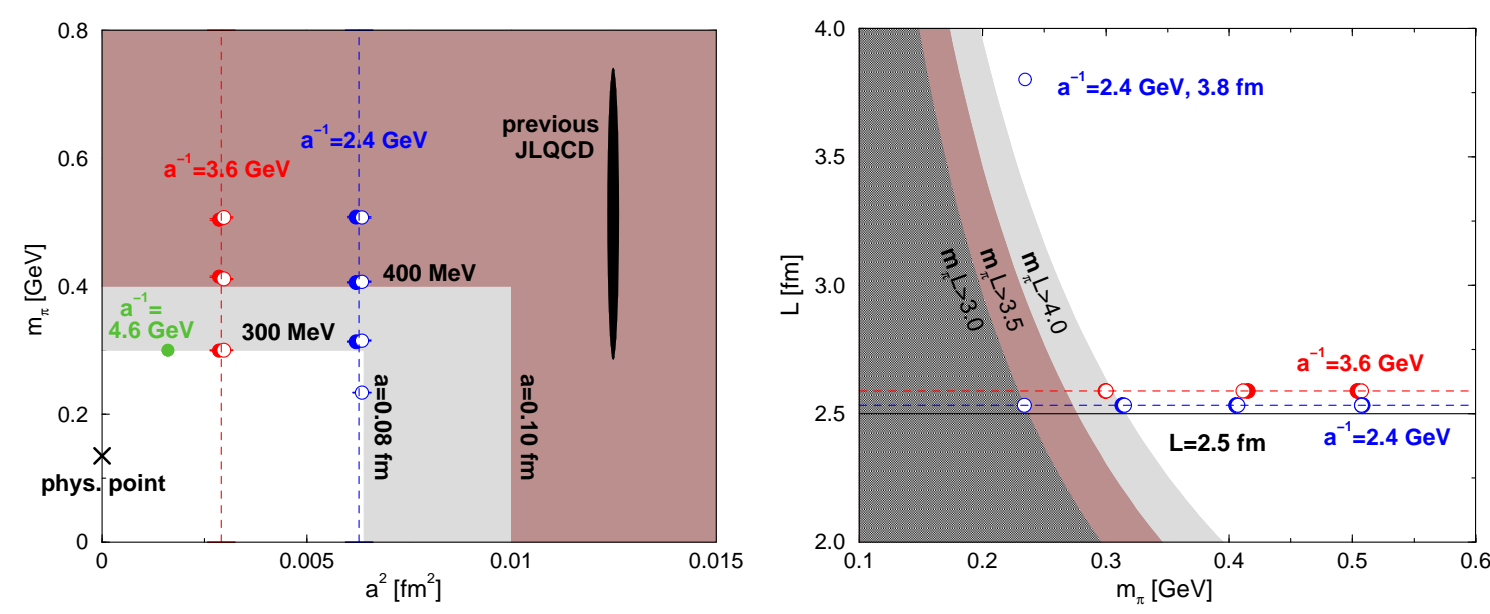

Figure 1: Overview of the simulation parameters. Left: simulation points in the $\left(a^{2}, m_{\pi}\right)$ plane. Two regions $\left(a>0.08 \mathrm{fm}, m_{\pi}>300 \mathrm{MeV}\right)$ and $\left(a>0.10 \mathrm{fm}, m_{\pi}>400 \mathrm{MeV}\right)$ are indicated by different colors. Right: Parameters in the $\left(m_{\pi}, L\right)$ plane. We show the regions of $m_{\pi} L \gtrsim 3.0,3.5$ and 4.0 by different colors. See the text for more details.

give an estimate of the finite size effects. We note that the length of the unit trajectory is increased from the standard choice $\tau=1$ to $\tau=2$ at $a^{-1} \approx 3.6 \mathrm{GeV}$ and $\tau=4$ at $4.6 \mathrm{GeV}$ in order to reduce the effects of possible long autocorrelations at these finer lattices [ [3]. Table $\square$ lists the parameters and the current statistics of these gauge ensembles. By using the Möbius kernel and three levels of the stout smearing, the Ginsparg-Wilson relation is precisely satisfied with a moderate size in the 5 -th direction of the lattice $L_{5}$. In fact, we obtain the residual mass at a level of $m_{\text {res }}<0.5 \mathrm{MeV}$ at $\beta=4.17$ and even smaller values $m_{\text {res }}<0.1 \mathrm{MeV}$ at $\beta=4.35$.

Parameters of the generated gauge configurations are summarized in the two panels of Figure $\mathrm{W}$. The left panel shows the simulation points in the $\left(a^{2}, m_{\pi}\right)$ plane. Using the physical scale as discussed in the next section, we plot the pion mass at $\beta=4.17$ (blue) and 4.35 (red) with filled (open) symbols for the lighter (heavier) strange quark mass. The point for $\beta=4.47$ (green) represents a temporary estimate. Note that we are exploring much finer lattices compared to our previous project which covers the black region in the left panel. The right panel shows the simulation points in the $\left(m_{\pi}, L\right)$ plane. All points with $m_{\pi} \gtrsim 300 \mathrm{MeV}$ satisfy the condition $m_{\pi} L \gtrsim 4.0$ for a good control of finite volume effects. Two points with $m_{\pi} \approx 240 \mathrm{MeV}$ at $\beta=4.17$ are carried out on two different physical volumes, $m_{\pi} L \approx 4.5$ and 3.0, for a direct check of the volume effects.

In the rest of this article we present some basic tests of the generated gauge configurations as a preparation for physics studies. For other related works, see [四, []].

\section{Scale setting by the Yang-Mills gradient flow}

We determine the physical scale by the Yang-Mills gradient-flow time $t_{0}$ which is defined by $\left.t^{2}\langle E\rangle\right|_{t=t_{0}}=0.3[\square] . E$ is the energy density of the gauge field calculated using its clover-leaf definition. Compared to the alternative way of using $w_{0}$ defined by $\left.t \frac{d}{d t}\left(t^{2}\langle E\rangle\right)\right|_{t=w_{0}^{2}}=0.3$ [ [ $]$ ], the scale setting by $t_{0}$ shows milder mass dependence and smaller statistical error as reported in [Q]. 

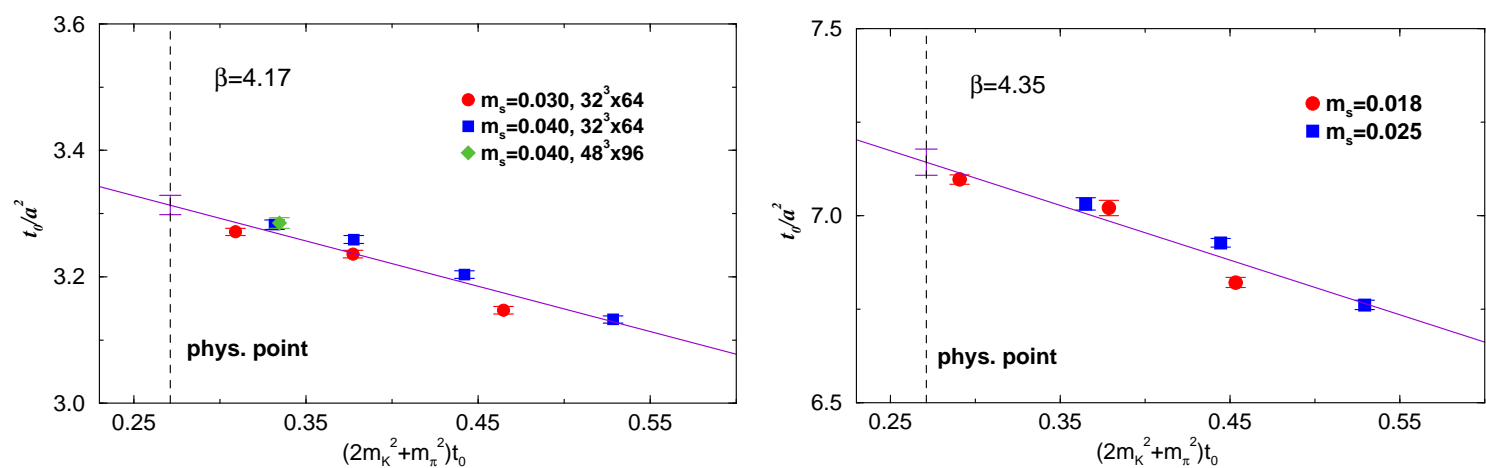

Figure 2: $t_{0} / a^{2}$ as a function of $\left(2 m_{K}^{2}+m_{\pi}^{2}\right) t_{0}$ for $\beta=4.17$ (left) and 4.35 (right). In each panel, the solid line indicates the linear fit to the data.
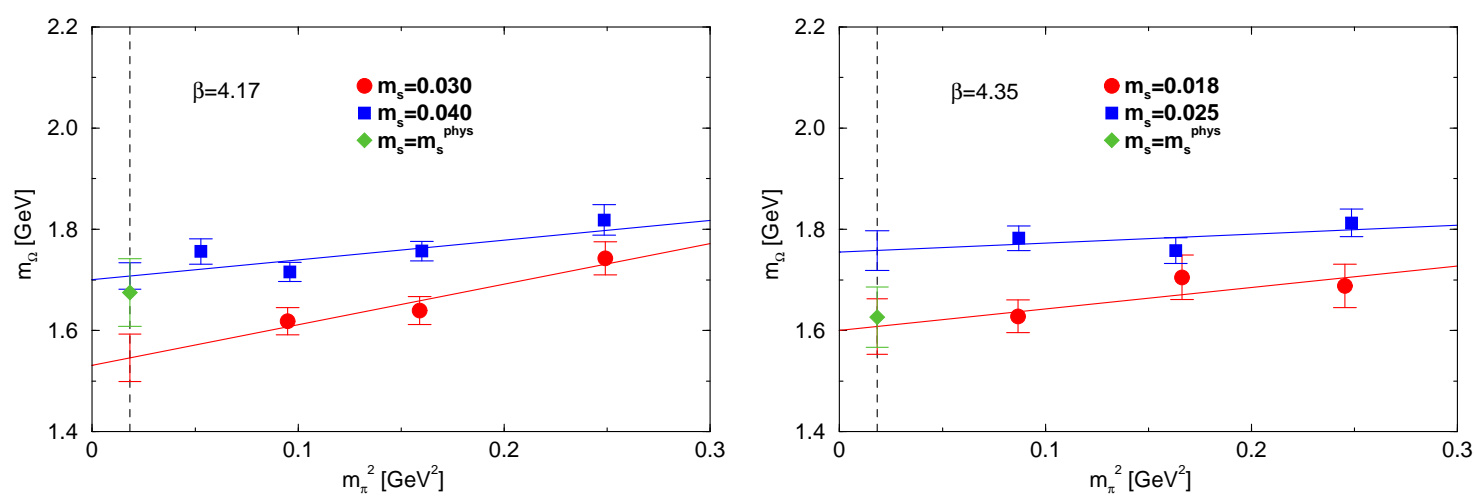

Figure 3: $m_{\Omega}$ as a function of $m_{\pi}^{2}$ in physical units at $\beta=4.17$ (left) and 4.35 (right). In each panel, the solid line indicates the linear fit to the data. The green diamonds represent the physical values obtained by the interpolation of $m_{s}$.

Chiral expansion of $t_{0} / a^{2}$ would take a form

$$
t_{0} / a^{2}=\left(\bar{t}_{0} / a^{2}\right)\left[1+\frac{k_{1}}{(4 \pi f)^{2} \bar{t}_{0}}\left(2 m_{K}^{2}+m_{\pi}^{2}\right) t_{0}+\mathscr{O}\left(M^{4}\right)\right],
$$

where $k_{1}, f$ and $\bar{t}_{0}$ (the value of $t_{0}$ in the chiral limit) are unknown parameters [ए0]]. $M$ represents a typical light meson energy-scale. Since there is no non-analytic contribution at the order of $M^{2}$, we carry out a linear extrapolation of $t_{0} / a^{2}$ as a function of $\left(2 m_{K}^{2}+m_{\pi}^{2}\right) t_{0}$, which is proportional to the total sea quark mass $2 m_{u d}+m_{s}$. For the physical point, we use $m_{\pi}=135 \mathrm{MeV}, m_{K}=495$ $\mathrm{MeV}$ and $t_{0}^{1 / 2}=0.1465 \mathrm{fm}$ as inputs [ [ 8$]$. The left and the right panels of Figure $\square$ show this chiral extrapolation at $\beta=4.17$ and 4.35 , respectively. At the physical point, we obtain

$$
a^{-1}= \begin{cases}2.45(1) \mathrm{GeV} & (\beta=4.17), \\ 3.60(2) \mathrm{GeV} & (\beta=4.35),\end{cases}
$$

where we quote only the statistical error. Analysis including higher order terms, which are visible as a difference between two $m_{s}$, is to be performed. We observe a good agreement in $t_{0} / a^{2}$ between the two different volumes (blue and green symbols) at $\left(m_{\mathrm{ud}}, m_{\mathrm{s}}\right)=(0.0035,0.0400)$ in the left panel. The systematic error from the finite volume size should therefore be small. 

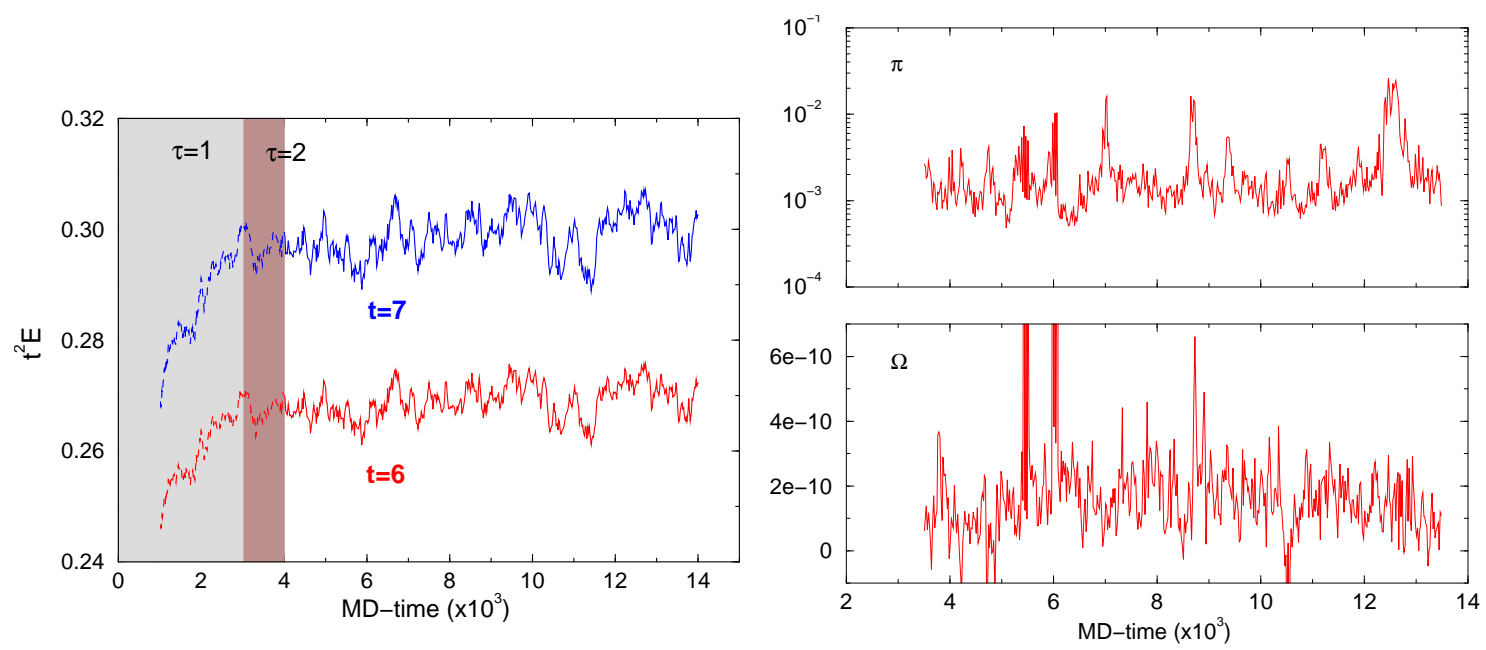

Figure 4: The Monte Carlo history of several quantities taken from the ensemble at $\beta=4.35$ and $\left(m_{\mathrm{ud}}, m_{\mathrm{s}}\right)=$ $(0.0042,0.0250)$. Left: history of $t^{2} E$ at $t=6$ (red) and 7 (blue). Both the gray $(\tau=1)$ and brown $(\tau=2)$ regions represent the thermalizing trajectories. Right: history of the point source correlators of pion (upper) and $\Omega$-baryon (lower) at a time slice $t=15$.

We also compute the Sommer scale $r_{0} / a$ on each ensemble and extrapolate the data to the physical point as a linear function of $\left(2 m_{K}^{2}+m_{\pi}^{2}\right) t_{0}$. From (L.2) and the extrapolated values of $r_{0} / a$, we obtain consistent values $r_{0}=0.469(4) \mathrm{fm}(\beta=4.17)$ and $0.45(1) \mathrm{fm}(\beta=4.35)$, where only the statistical errors are quoted. These estimates are reasonably consistent with $r_{0}=0.45-0.50 \mathrm{fm}$ obtained so far in 2+1-flavor QCD [Q]

We also attempt a scale determination from the $\Omega$-baryon mass. As mentioned in the previous section, we have two gauge ensembles with different strange quark masses, $m_{s}^{\mathrm{h}}>m_{s}^{1}$, at each $\beta$. We first estimate the $\Omega$-baryon mass $m_{\Omega}^{\mathrm{h}}\left(m_{\Omega}^{1}\right)$ at $m_{s}^{\mathrm{h}}\left(m_{s}^{\mathrm{l}}\right)$ and at the physical pion mass $m_{\pi}^{2}=$ $(135 \mathrm{MeV})^{2}$ by assuming a linear fitting function in terms of $m_{\pi}^{2}$. Left and right panels of Figure [] show these plots for $\beta=4.17$ and 4.35 , respectively. Linear combination $m_{\eta_{s}}^{2}=2 m_{K}^{2}-m_{\pi}^{2}$ is extrapolated to the physical pion mass and resulting values, $\left(m_{\eta_{s}}^{2}\right)^{1}$ and $\left(m_{\eta_{s}}^{2}\right)^{\mathrm{h}}$, are used to tune the strange quark mass. We then estimate $m_{\Omega}$ at the physical point by

$$
m_{\Omega}^{\text {phys }}=m_{\Omega}^{1}+\frac{\left(m_{\eta_{s}}^{2}\right)^{\text {phys }}-\left(m_{\eta_{s}}^{2}\right)^{1}}{\left(m_{\eta_{s}}^{2}\right)^{\mathrm{h}}-\left(m_{\eta_{s}}^{2}\right)^{1}} \cdot\left(m_{\Omega}^{\mathrm{h}}-m_{\Omega}^{1}\right)
$$

Our estimates $m_{\Omega}^{\text {phys }}=1.67(7) \mathrm{GeV}(\beta=4.17)$ and 1.63(6) $\mathrm{GeV}(\beta=4.35)$ with $m_{\eta_{s}}^{\text {phys }}=a^{2}[2$. $\left.(495 \mathrm{MeV})^{2}-(135 \mathrm{MeV})^{2}\right]$ are consistent with the experimental value $m_{\Omega}=1.672 \mathrm{GeV}$.

These observations indicate that we obtain consistent estimates of the lattice scale $a^{-1}$ from $t_{0}, r_{0}$ and $m_{\Omega}$.

\section{Thermalization and autocorrelation}

As already reported in [四, we found a long thermalization time for the finer lattices at $\beta=$ 4.35. It gives a warning that the thermalization and autocorrelation should be monitored and checked for various quantities covering both gluonic and fermionic observables. Observables from 

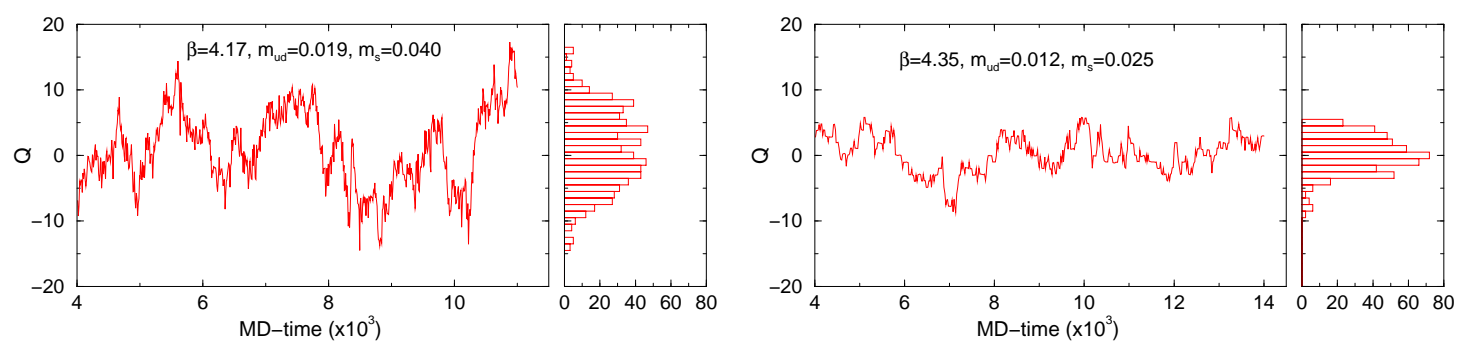

Figure 5: The Monte Carlo history and the histogram of the topological charge $Q$ at $\beta=4.17$ (left) and and 4.35 (right). Examples with the heaviest quarks are shown for each $\beta$.
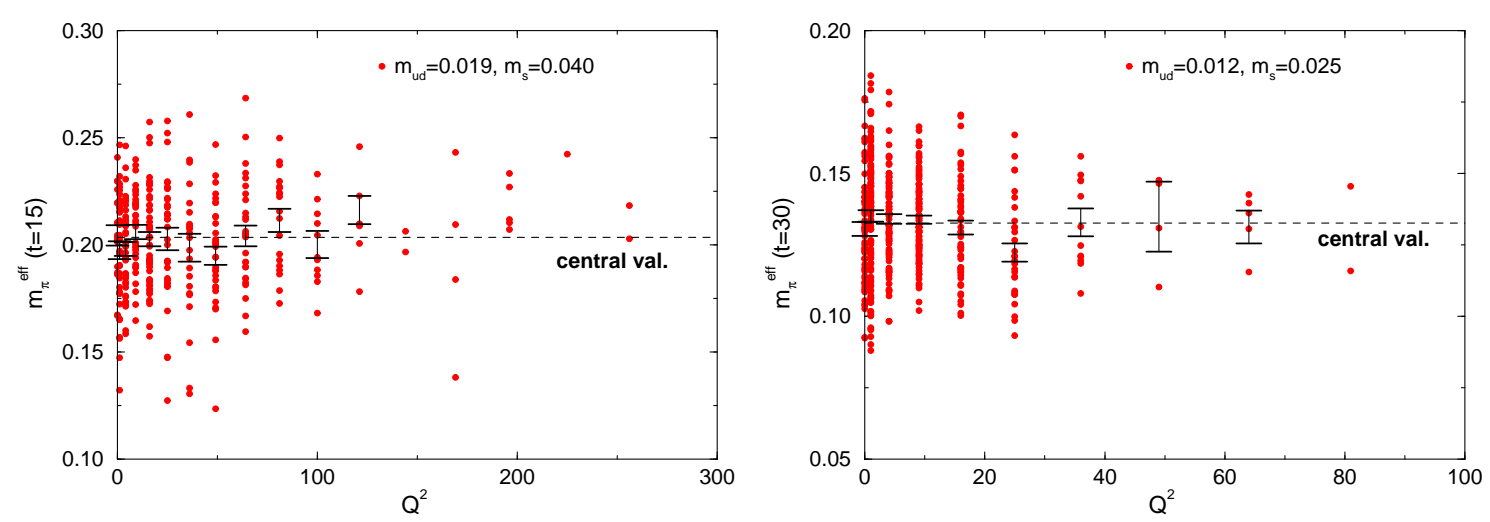

Figure 6: Scatter plots of the pion effective mass versus $Q^{2}$ for $\beta=4.17$ (left, at $t=15$ ) and 4.35 (right, at $t=30$ ). The central value over the entire data is shown with a dashed line. On several topological sectors, central values and statistical errors are also shown. Examples from the same ensembles as Figure 4.

the Yang-Mills gradient flow are particularly useful because they are statistically very precise and have a sensitivity to the IR fluctuations, for which the long autocorrelation is expected. The left panel of Figure $\rightarrow$ shows the history of $t^{2} E$ at the flow time $t / a^{2}=6$ and 7 , around which $t_{0}$ is extracted. We observe that HMC undergoes thermalization during the first 4,000 MD-time. After that, the gauge configurations do not seem to be trapped in any specific region. A quantitative analysis of the autocorrelation time is ongoing.

In the right panel of Figure $\$$, we also check the autocorrelation of hadron correlators. Compared to the similar plots for $t^{2} E$, the autocorrelation is less significant for these hadronic observables.

\section{Global topological charge and correlation with hadronic observables}

Another but related concern in the ensemble generation is the nearly frozen topology at the finer lattice spacings. We evolve the stored gauge configurations by the Yang-Mills gradient flow and compute the topological charge $Q=\frac{1}{16 \pi^{2}} \sum_{x} \operatorname{tr} F_{\mu v}(x) \tilde{F}_{\mu v}(x)$. We extract a measurement of $Q$ after the measured value comes to a plateau in $t$. From the history of $Q$, we confirm that a reasonable sampling of different topological sectors is obtained for each ensemble of $\beta=4.17$ and 4.35. Figure $\square$ shows the examples of the MC history and the histogram from the heaviest quark mass ensembles at each $\beta$. The figure shows a significantly narrower distribution of $Q$ at $\beta=4.35$ 
(right two panels) than $\beta=4.17$ (left two panels), though they share similar values of $m_{\mathrm{ud}}, m_{\mathrm{s}}$ and the physical volume. This suggests that the global topological charge $Q$ decorrelates more slowly on the finer lattice. In [目], we propose a method to extract correct physical results in such situations and present the results for the topological susceptibility.

For a precision study of hadron physics, it is desirable to check possible influences of the global topology freezing to hadronic observables. Figure 6 shows scatter plots of the pion effective mass $m_{\pi}^{\text {eff }}$ versus $Q^{2}$ at $\beta=4.17$ (left) and 4.35 (right). We observe that the correlation between $Q$ and the distribution of $m_{\pi}^{\text {eff }}$ is negligible. Actually, averages of $m_{\pi}^{\text {eff }}$ in different topological sectors are in good agreement with that over all the sectors. This observation together with an analytic study of the effects of fixing topology [U]] implies that the influences of the slow fluctuation of $Q$ to hadronic observables are not significant in our simulated region of the lattice spacing.

Numerical simulations are performed on the IBM System Blue Gene Solution at High Energy Accelerator Research Organization (KEK) under a support of its Large Scale Simulation Program (No. 13/14-04). This work is supported in part by the Grant-in-Aid of the Japanese Ministry of Education (Nos. 21674002, 25287046, 25800147, 26400259) and the SPIRE (Strategic Program for Innovative Research) Field5 project.

\section{References}

[1] J. Noaki, S. Aoki, G. Cossu, H. Fukaya, S. Hashimoto and T. Kaneko, Fine lattice simulations with chirally symmetric fermions, PoS(LATTICE 2013) 263.

[2] G. Cossu, J. Noaki, S. Hashimoto, T. Kaneko, H. Fukaya, P.A. Boyle and J. Doi, JLQCD IroIro++ lattice code on BG/Q, PoS(LATTICE 2013) 482, URL:

http://suchix.kek.jp/guido_cossu/documents/DoxyGen/html/index.html

[3] H. Mayer et al. (Alpha Collaboration), Exploring the HMC trajectory-length dependence of autocorrelation times in lattice QCD, Comp. Phys. Comm. 176 (2007) 91.

[4] Y-G. Cho, S. Hashimoto, A. Jüttner, T. Kaneko, M. Marinkovic, J. Noaki and J-T. Tsang, Scaling study of an improved fermion action on quenched lattices, in these proceedings, pp381.

[5] M. Tomii, G. Cossu, S. Hashimoto and J. Noaki, Nonperturbative renormalization of bilinear operators with Mobius domain-wall fermions in the coordinate space, in these proceedings, pp287.

[6] H. Fukaya, S. Aoki, G. Cossu, S. Hashimoto, T. Kaneko and J. Noaki, Topology density correlator on dynamical domain-wall ensembles with nearly frozen topological charge, in these proceedings, pp323.

[7] M. Lüscher, Properties and uses of the Wilson flow in lattice QCD, JHEP 08 (2010) 071.

[8] S. Borsanyi et. al. (BMW Collaboration), High-precision scale setting in lattice QCD, JHEP 09 (2012) 010 .

[9] R. Sommer, Scale setting in lattice QCD, PoS(LATTICE 2013) 015.

[10] O. Bar and M. Golterman, Chiral perturbation theory for gradient flow observables, Phys. Rev. D 89 (2014) 034505.

[11] S. Aoki, H. Fukaya, S. Hashimoto and T. Onogi, Finite volume QCD at fixed topological charge, Phys. Rev. D 76 (2007) 054508. 